\title{
National Center for Complementary and Alternative Medicine The Role of Complementary, Alternative, and Integrative Medicine in Personalized Health Care
}

\author{
Emmeline Edwards ${ }^{*, 1}$ \\ ${ }^{1}$ Division of Extramural Research, National Center for Complementary and Alternative Medicine, National Institutes of Health, \\ Bethesda, MD, USA
}

Neuropsychopharmacology Review (2012) 37, 293-295; doi: I0.1038/npp.20II.92

Personalized medicine, the treatment of patients based on their individual genetic, epigenetic, and phenotypic make up, holds the promise of dramatically changing healthcare by allowing clinical researchers to find specific and more effective strategies to manage symptoms of chronic diseases. Personalized medicine also involves the development of treatment strategies that incorporate understanding of individual patient preference. In a pluralistic healthcare system like that in the United States, many patients explore health practices that are not part of mainstream medicine. The National Center for Complementary and Alternative Medicine (NCCAM/NIH) has sponsored two national surveys as components of the National Health Interview Survey (NHIS) of the centers for disease control and prevention. These surveys have shown that nearly $40 \%$ of adult Americans and $12 \%$ of children used some form of complementary or alternative medicine as a complement or adjunct to conventional care (Barnes et al, 2008). Examples include use of natural products, such as herbal medicines and probiotics, and mind and body practices, such as meditation, progressive relaxation, tai chi, yoga, acupuncture, massage therapy, and spinal manipulation. In the last decade, as evidence for some of these approaches has grown, they are increasingly being incorporated into conventional care as part of an integrative medicine approach. An understanding of the biological basis of these CAM interventions and an increasing knowledge of how CAM treatments impact disease processes in specific individuals and populations could contribute to the development of more personalized strategies for the management of chronic conditions and the support of health promoting behaviors.

${ }^{*}$ Correspondence: $\operatorname{Dr}$ E Edwards, Division of Extramural Research, National Center for Complementary and Alternative Medicine, National Institutes of Health, 6707 Democracy Boulevard, Suite 401, Bethesda, MD 20892-5475, USA, Tel: +301 496 4792, Fax: +301 4801587 , E-mail: edwardse@mail.nih.gov

Received 31 March 2011; revised 15 April 2011; accepted 22 April 2011
The newly released NCCAM third strategic plan, Exploring the Science of Complementary and Alternative Medicine, emphasizes the need to improve the evidence base for the use of CAM in treating health problems and promoting health and well being. NCCAM supports research to advance the science and practice of symptoms management, and to develop effective, practical, and personalized strategies for promoting health and well being. This commentary will limit its focus on three areas with high scientific promise and potential for interdisciplinary and integrative research - management of chronic pain, management of mood and stress-related disorders, and management of substance abuse/behavioral addiction.

\section{SYMPTOM MANAGEMENT IN CHRONIC PAIN}

Chronic pain is a huge public-health problem affecting $~ 25 \%$ of adults. Often these individuals seek relief from a variety of approaches including opioids, and invasive interventions, such as nerve blockade and surgery. Increasingly it is acknowledged that more research is needed on non-pharmacological approaches including physical therapy, as well as a number of CAM therapies including spinal manipulation, yoga, exercise therapy, acupuncture, massage, mindfulness-based stress reduction, and cognitive behavioral therapy. CAM approaches show promise for a variety of benefits including changes in pain perception and processing, increased cognitive control, improved coping skills, decreased pain intensity, and increased well being and relaxation. In implementing its stated goal of 'Advancing the Science and Practice of Symptom Management', NCCAM's researchers are applying state-of-theart technologies to elucidate musculoskeletal biomechanics, central nervous system responses, emotional and cognitive influences, and behavior and genetics in pain management.

The NCCAM clinical research portfolio includes the development of improved strategies for managing chronic 
pain studies promoting a better understanding of natural history and prognostic factors, the development of improved diagnostic criteria and tools, and the increased utilization of pragmatic trials and outcomes research. NCCAM is supporting several clinical studies that are aimed at identifying biomarkers for pain prognosis and treatment response. Many healthcare settings have positively tested the effectiveness of non-pharmacological and bio-psychosocial management of chronic pain (Bercovitz et al, 2011; Smeeding et al, 2010). Other promising research avenues include the use of placebo, with informed consent as an effective treatment for pain conditions, and the characterization of psychological (suggestibility, optimism, and expectation) and neurobiological (opioid system activation, reward responses, and gray matter density) correlates of individual differences in placebo responses (Wager et al, 2011).

\section{INTEGRATIVE APPROACHES FOR MOOD DISORDERS}

With a better appreciation of the genetic complexity of mood disorders, there is some evidence that multiple genes may exert an effect in concert with non-genetic factors to produce a risk of mental disorder. However, the promise that genetics would yield new therapies that aimed at pathogenic processes and symptoms management is not fully realized. Treatments are needed that can complement and add to the benefits of pharmacotherapy, thus permitting the targeting of specific therapies to individual patients.

One recent study suggests that mindfulness-based cognitive therapy (MBCT), a combination of cognitive behavioral therapy with daily practice of meditation, yoga, and other health-enhancing behaviors can be as effective as long-term antidepressant medication alone in preventing relapse in patients who have had several bouts of major depression (Segal et al, 2010). The biological factors associated with mindfulness meditation may include changes in brain function that can be documented using electrophysiology, single-photon emission-computed tomography, PET, and functional magnetic resonance imaging (Slagter et al, 2011). NCCAM researchers are exploring the associations of major pathways of cognitive processing and emotion regulation by meditative practices. Although speculative at this stage, the identification of biological markers of these practices may foster the convergence of the biological and psychological aspects of psychiatry, and also aid in the design of primary prevention studies in mood disorders.

Many individuals suffering from depression and anxiety are using natural products to manage symptoms and improve quality of life. Definitive evidence of efficacy for most of these interventions does not exist. NCCAM supported one large trial on St John's Wort (Hypericum perforatum) for major depression, which failed to demonstrate benefit. Both pre-clinical studies using established animal models of depression and anxiety, as well as clinical studies, are considered areas of active interest and of some

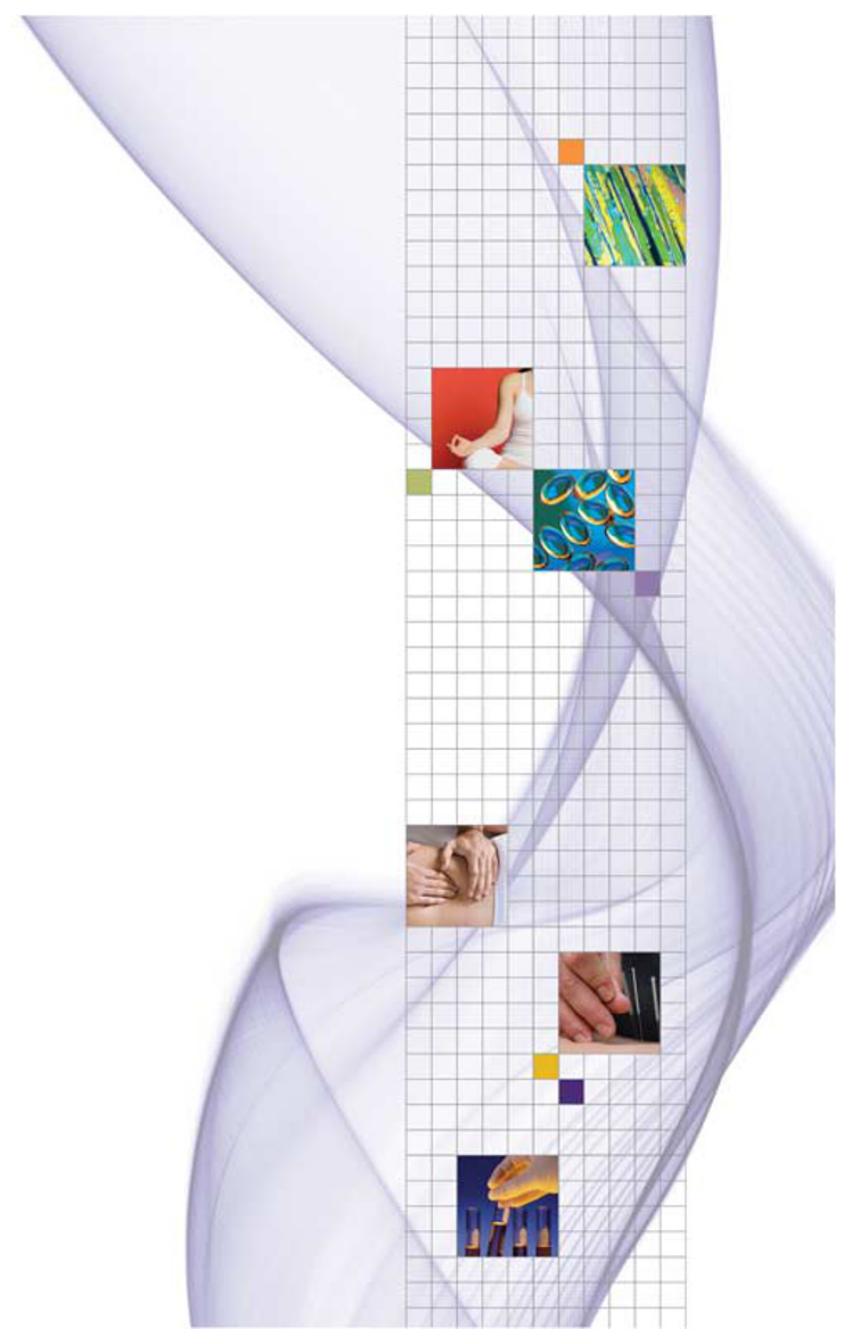

Figure 1. Complementary and alternative medicine modalities and therapies.

potential. NCCAM is actively encouraging mechanistic studies to elucidate the biological effects, mechanisms of action, and safety profiles of CAM natural products, laying the ground work or the solid biological foundation for translational research needed to carry out clinical studies.

\section{SUBSTANCE ABUSE/BEHAVIORAL ADDICTION}

A growing body of research suggests that interventions that help individuals to cope better with stress, negative affect, and trauma, may offer complementary and alternative strategies in substance abuse treatment. For example, the practice of yoga, which has been generally linked to reductions in stress, anxiety, and increased self-efficacy, was shown to help reduce the negative symptoms that often accompany smoking cessation and predict relapse among recent quitters (Bock et al, 2010). Mindfulness training also provides an alternative and targeted treatment for stressprecipitated alcohol dependence among vulnerable members of society. 
A stated objective in NCCAM's new strategic plan is to increase the understanding of 'real-world' patterns and outcome of CAM use and integration into healthcare systems. Mind-body interventions in relapse prevention are of increasing clinical and scientific interest, but they also present opportunities to implement relatively low-risk, lowcost, and acceptable treatment options in community care settings and practice-based research networks.

\section{FUTURE DIRECTIONS}

Studies of how to effectively incorporate the patient's experiences and preferences into the health encounter, better understanding of how interactions between patient and provider influence health behaviors and their impact on health conditions co-morbid to pain, depression, and substance abuse, are important areas of research for NCCAM (http://nccam.nih.gov/about/plans/2011/ (Link to NCCAM 2011-2015 strategic plan)). The development of an improved evidence-base for personalized, and patientdecision making about the use of complementary and alternative medicine is a guiding principle for NCCAM's research investments.

\section{DISCLOSURE}

The author declares no conflict of interest.

\section{REFERENCES}

Barnes PM, Bloom B, Nahin RL (2008). Complementary and alternative medicine use among adults and children: United States 2007. Natl Health Stat Report 12: 1-23. This report provides national estimates on the use of complementary and alternative medicine in adults and children.

Bercovitz A, Sengupta M, Jones A, Harris-Kojetin LD (2011). Complementary and alternative therapies in hospices: the national home and hospice care survey: United States, 2007. Natl Health Stat Reports 33: 1-20. National estimates on the provision and use of complementary and alternative therapies (CAT) in hospices.

Bock BC, Morrow KM, Becker BM, Williams DM, Tremont G, Gaskins RB et al (2010). Yoga as a complementary treatment for smoking cessation: rationale, study design and participant characteristics of the Quitting-in-Balance Study. BMC Complement Altern Med 10: 14-20. This pilot efficacy study designed to examine the rates of cessation among women shows yoga to be a more effective complementary treatment for smoking cessation than traditional aerobic exercise.

Segal ZV, Bieling P, Young T, MacQueen G, Cooke R, Martin L et al (2010). Antidepressant monotherapy vs. sequential pharmacotherapy and mindfulness based cognitive behavioral therapy, or placebo for relapse prophylaxis in recurrent depression. Arch Gen Psychiatry 67: 1256-1264. Mindfulness-based cognitive therapy shows promise in preventing recurrent depression.

Slagter HA, Davidson RJ, Lutz A (2011). Mental training as a tool in the neuroscientific study of brain and cognitive plasticity. Front. Hum. Neurosci 5 : 17-23. The authors provide a working definition of meditation and discuss key findings from various methodological advances that characterize the biological factors underlying meditation training.

Smeeding SJ, Bradshaw DH, Humpfer K, Trevithick S, Stoddard GJ (2010). Outcome evaluation of the Veterans Affairs Salt Lake City Integrative Health Clinic for chronic pain and stress-related depression, anxiety, and post-traumatic stress disorder. J Altern Complement Med 16: 823-835. IHCP is an effective program, improving chronic pain and stress-related depression, anxiety, and health-related quality of life.

Wager TD, Atlas LY, Leotti LA, Rilling JK (2011). Predicting individual differences in placebo analgesia: contributions of brain activity during anticipation and pain experience. J Neurosci 31: 439-452. Engagement of emotional appraisal circuits drives individual variation in placebo analgesia, rather than early suppression of nociceptive processing. 